\title{
Release of the Styryl Dyes from Single Synaptic Vesicles in Hippocampal Neurons
}

\author{
Xi Chen, ${ }^{1}$ Sebastian Barg, ${ }^{2}$ and Wolfhard Almers ${ }^{1}$ \\ ${ }^{1}$ Vollum Institute, Oregon Health \& Sciences University, Portland, Oregon 97239, and ²Department of Cell Biology, Division of Medicine, Sir Alexander \\ Fleming Building, Imperial College London, London SW7 2AZ, United Kingdom
}

In small presynaptic boutons in brain, synaptic vesicles are thought not to merge with the plasma membrane when they release transmitter, but instead to close their fusion pores and survive intact for future use (kiss-and-run exocytosis). The strongest evidence for this idea is the slow and incomplete release of the fluorescent membrane marker, FM1-43 [N-(3-triethylammoniumpropyl)-4-(4(dibutylamino)styryl) pyridinium dibromide], from single vesicles. We investigated the release of FM1-43 from sparse cultures of hippocampal neurons grown on coverslips with no glia. This allowed presynaptic boutons to be imaged at favorable signal-to-noise ratio. Sparingly stained boutons were imaged at high time resolution, while high-frequency electrical stimulation caused exocytosis. The release of FM1-43 was quantal and occurred in abrupt steps, each representing a single fusion event. The fluorescence of vesicle clusters traveling along axons had a distribution with the same quantal size, indicating that a vesicle releases all the dye it contains. In most fusion events, the time constant of dye release was $<100 \mathrm{~ms}$, and slower release was rarely observed. After exocytosis, no FM1-43 could be detected in the axon to either side of a bouton, indicating that dye was released before it could spread. Our results are consistent with synaptic vesicles fusing fully with the plasma membrane during high-frequency stimulation.

Key words: presynaptic terminal; quantal release; fluorescence microscopy; vesicle transport; kiss-and-run; hippocampus

\section{Introduction}

Neurons package neurotransmitter into synaptic vesicles and release it when the vesicles fuse with the plasma membrane during exocytosis. The membrane of the vesicles then becomes exposed to the outside of the cell, is retrieved by endocytosis, and ultimately recycled to make new synaptic vesicles (Miller and Heuser, 1984; Takei et al., 1996). Styryl dyes such as FM1-43 [N-(3-triethylammoniumpropyl)-4-(4-(dibutylamino)styryl) pyridinium dibromide] have been widely used to investigate synaptic vesicle recycling (Betz and Bewick, 1992; Cochilla et al., 1999). They report endocytosis when synaptic terminals bathed in FM1-43 sequester the dye into endocytic vesicles (Ryan et al., 1993; Richards et al., 2000), and they report exocytosis when stimulated neurons destain as they release the dye along with the neurotransmitter (Betz and Bewick, 1993; Ryan et al., 1993). Despite some shortcomings, styryl dyes have a unique advantage over genetically targeted optical labels such as synaptopHluorin (Sankaranarayanan and Ryan, 2000) and synaptophysin-pHluorin (Granseth et al., 2006). By applying them only briefly, or during periods when little endocytosis occurs (Ryan et al., 1997), one may stain one or few among the 100-200 synaptic vesicles in the presynaptic bouton of a hippocampal neuron. When such mini-

Received 0ct. 3, 2007; revised Dec. 13, 2007; accepted Jan. 5, 2008.

This work was supported by National Institutes of Health Grant MH60600. We thank Barbara Smoody and Dr. Gary Banker for advice with hippocampal cultures and Drs. Steve Arch and William Betz for their comments on this manuscript.

Correspondence should be addressed to Wolfhard Almers, Vollum Institute, Oregon Health \& Sciences University, 3181 Southwest Sam Jackson Park Road, Portland, OR 97239. E-mail: almersw@ohsu.edu.

DOI:10.1523/JNEUROSCI.4518-07.2008

Copyright $\odot 2008$ Society for Neuroscience $\quad$ 0270-6474/08/281894-10\$15.00/0 mally stained boutons are stimulated exhaustively, the total amount of dye released by each showed a quantal distribution (Ryan et al., 1997; Murthy and Stevens, 1998; Aravanis et al., 2003; Krueger et al., 2003; Lemke and Klingauf, 2005). The quantum can represent either the single endocytic event or the dye released during the exocytosis of a single synaptic vesicle or both. In fish retinal bipolar neurons, the exocytosis of single synaptic vesicles could be observed by total internal reflection fluorescence (TIRF) microscopy (Zenisek et al., 2000; Midorikawa et al., 2007), but similarly direct imaging of exocytosis in mammalian neurons has been more difficult.

Is the release of dye in hippocampal neurons quantal, as it is for neurotransmitter from motor neurons? Does a synaptic vesicle release all its dye in a single exocytic event? Murthy and Stevens (1998) suggested that the answer is yes. However, when this point was later tested in time-resolved recordings, single fusions did not generally cause quantal dye release, and synaptic vesicles released only a portion of the dye within them (Aravanis et al., 2003; Richards et al., 2005). The results suggests "kiss-andrun" and differ from the all-or-nothing release expected if vesicles flatten into the plasma membrane after exocytosis (Heuser and Reese, 1973). A debate continues as to whether kiss-and-run predominates in hippocampal or other neurons (Granseth et al., 2006; Zhang et al., 2007).

Much may be gained if the exocytosis of synaptic vesicles could be recorded at better signal-to-noise ratio and higher time resolution. Here we have used a culture system in which neurons and glia grew separately (Banker and Goslin, 1998), and the background fluorescence was diminished. This allowed us to record dye release from single vesicles while boutons were stimulated at 


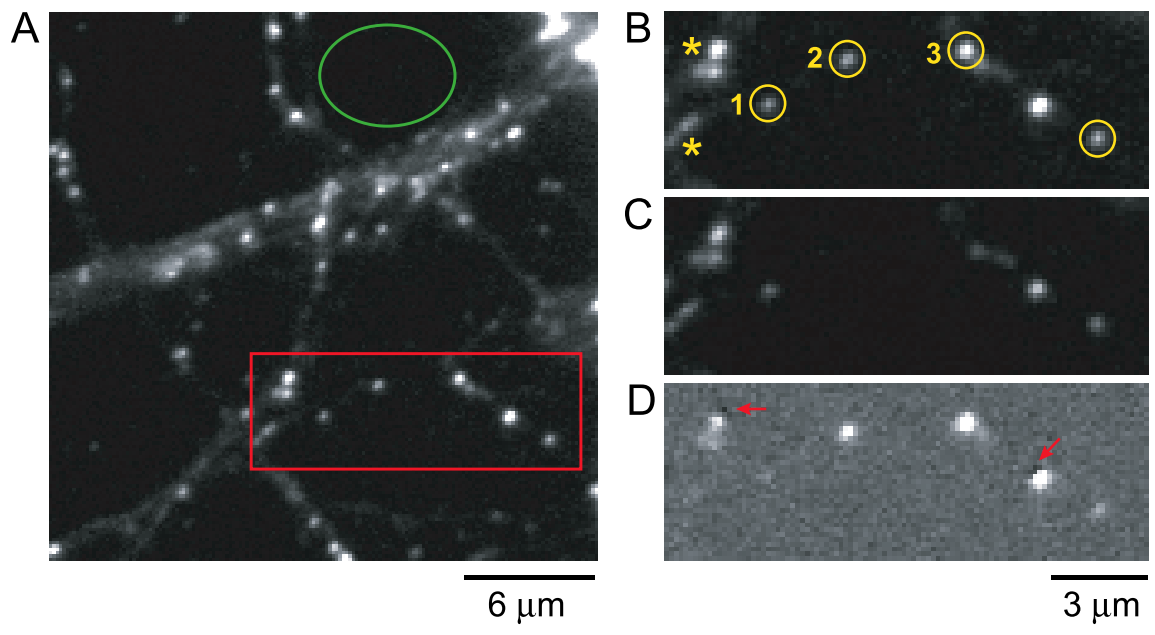

Figure 1. Minimal staining of sparse hippocampal cultures. A, Fluorescence image of a hippocampal culture minimally stained with FM1-43. A thick fascicle and numerous smaller ones are seen to surround extensive cell-free areas; one such area is outlined in green. The image shows $\sim 10 \%$ of the field of view and is an average of 30 images taken in the last $1 \mathrm{~s}$ before the onset of field stimulation. $\boldsymbol{B}$, Magnified version of the square outlined in red in $\boldsymbol{A}$. Circles indicate primary regions selected for additional analysis. Regions 1,2 , and 3 correspond to traces $\boldsymbol{E}, \boldsymbol{F}$, and $\boldsymbol{I}$ in Figure 2 . $\boldsymbol{C}$, Average of 30 images taken in the $1 \mathrm{~s}$ after the last shock in a train of shocks. The structures marked in $\boldsymbol{B}$ have disappeared or are much dimmer. $\boldsymbol{D}, 0$ ne thousand fluorescence units were added to $\boldsymbol{B}$, and then $\boldsymbol{C}$ was subtracted. Arrows mark regions that gained fluorescence attributable to movement. All images except $\boldsymbol{D}$ are at the same contrast.

high frequency. As in retinal bipolar neurons, the release of dye occurred in tens of milliseconds and went to completion in a single fusion event.

\section{Materials and Methods}

Hippocampal neurons in CA1 and CA3 regions of embryonic day 18 rat embryos were plated onto 25 -mm-diameter coverslips and cultured for $14-28 \mathrm{~d}$ at low density in glia-conditioned medium (Banker and Goslin, 1998). This produced coverslips containing neurons but no glial cells. For imaging, coverslips were mounted in a modified Tyrode's solution ( $149 \mathrm{~mm} \mathrm{NaCl}, 2.5 \mathrm{~mm} \mathrm{KCl}, 2 \mathrm{~mm} \mathrm{CaCl}, 2 \mathrm{~mm} \mathrm{MgCl}$, 30 mm glucose, 10 mM HEPES, $10 \mu \mathrm{M}$ CNQX, and $50 \mu \mathrm{m}$ APV, pH7.4) in a closed imaging chamber with embedded platinum wires for field stimulation (model P-2; Warner Instruments, Hamden, CT). Presynaptic boutons were stained by perfusing the chamber for $60 \mathrm{~s}$ with a Tyrode's solution containing styryl dye in the absence of stimulation. They were then washed in dye-free Tyrode's solution for $15 \mathrm{~min}$ (solution change $90 \%$ complete in $10 \mathrm{~s}$ ), and finally the microscope was focused in fewer than five exposures of $200 \mathrm{~ms}$ each. A single movie was recorded from each coverslip (100-300 frames at $28 \mathrm{~Hz}$ unless indicated otherwise), and field stimulation was applied (100 shocks at $100 \mathrm{~Hz}$ unless indicated otherwise). Usually, an image taken with differential interference contrast (DIC) concluded the experiment.

We used a Zeiss (Oberkochen, Germany) Axiovert S100 microscope with a Plan-Apo $63 \times / 1.4$ objective and a $1.6 \times$ Optovar magnifier. Epifluorescence excitation was by a XBO $75 \mathrm{~W}$ xenon arc lamp through an excitation filter $(450-490 \mathrm{~nm})$ using a 460 dichroic longpass mirror (cut-on wavelength of $460 \mathrm{~nm}$ ); the emission filter passed 520-650 nm. Mirrors and filters were from Chroma Technology (Brattleboro, VT). Images were taken with a Cascade 512B EM-CCD camera (PhotoMetrics, Tucson, AZ) at the following settings: digital gain 3, electron multiplication gain 3808 , stream acquisition. One pixel represented $159 \mathrm{~nm}$. A digital output from the camera opened the arc lamp shutter and triggered a square-wave generator that delivered field stimulation to the chamber ( $10 \mathrm{~V}, 2 \mathrm{~ms}$ pulses). The camera was run using MetaMorph (Universal Imaging, Downingtown, PA). All recordings were done at $27-28^{\circ} \mathrm{C}$. Means \pm SE are given.

Finding boutons. The last 30 frames before the first shock were averaged into a single prestimulus image. Because the cultures were sparse, typical images showed between zero and three cell bodies. Fluorescent spots representing putative presynaptic boutons were located in that image using the MetaMorph routine "Find Spots" (region size, $1.27 \mu \mathrm{m}$ ). The application found first the brightest and then progressively dimmer spots until the remaining spots failed to meet a brightness criterion ("spot cutoff," typically 100 arbitrary units). All spots were then examined visually in magnified portions of the image. Spots were rejected if they were unlikely to represent single and stationary synaptic vesicles, i.e., if they were blurred, too bright, oblong, or too large to be diffraction limited. If the centers of any two spots were within $1.27 \mu \mathrm{m}$ of each other, then both were rejected. We tested for movement in a difference image formed by averaging the first 30 frames starting $200 \mathrm{~ms}$ after the last stimulus and subtracting the result from the prestimulus image. To avoid negative pixels, a constant was added to the prestimulus image before the subtraction. Regions that had survived the selection described above were transferred into the difference image, and those with a dark "shadow" close by (arrows in Fig. $1 D$ ) were removed. The remaining regions were returned into the prestimulus image and repositioned slightly. In practice, difference and prestimulus images were reexamined iteratively until all regions were centered precisely on spots that were crisp, small, solitary, and far removed from shadows. Some 20-200 regions in each field of view (10-30\%) survived the selection procedure and comprised our set of primary regions marking putative single boutons. The selection required judgment on the part of the observer, but it was bias-free insofar as the observer had not seen the movies, and no additions or deletions were made during later stages in the analysis.

Background and bleaching. The primary regions were placed onto the movies. The average fluorescence in each was measured as a function of time with the "Graph Intensities" application in MetaMorph and placed into preprogrammed Excel (Microsoft, Seattle, WA) worksheets that completed the analysis. Each column in the worksheet contained a "trace" plotting the region's intensity against time during the movie (see Fig. 2A,B). Next, some 10-20 background regions were placed into the prestimulus image in places without neurites or cell bodies (Fig. 1A, green). Their fluorescence was measured as a function of time; the results were averaged (see Fig. 2C) and used to correct the fluorescence from the primary regions. Unless indicated otherwise, boutons with an initial brightness larger than that of fluorescein-coated beads (see below) were excluded from additional analysis because they were likely to have many more than one stained vesicle and because their fluorescence traces were noisy.

We used bright excitation light and accepted bleaching by $20-50 \%$ in a 300-frame movie. Our correction for bleaching was based on control experiments without field stimulation. Boutons were found and analyzed as above, background was subtracted, and the traces from each coverslip were averaged and scaled to an initial value of 1 . The rate of bleaching varied significantly from one culture to the next and, to some extent, even from coverslip to coverslip, possibly because the excitation intensity varied or for other unknown reasons. A sum of two exponentials was required to describe bleaching, as if each coverslip contained both rapidly and more slowly bleaching dye molecules. We assume that, although the rate of bleaching varies from one coverslip to the next, the underlying time course does not. In other words, if the fast-bleaching molecules in one coverslip bleach faster than in another, then the slow-bleaching molecules will bleach faster by the same factor. This assumption is appropriate, for example, if variations in bleaching rate were attributable to variations in the excitation intensity. In practice, the procedure was as follows. A single-exponential function was fitted to the averaged data from each control coverslip to obtain a rough measure of the time con- 
stant of bleaching. When the data were replotted against time measured in multiples of that time constant, the results from different cultures agreed well with one another. They were combined and fitted with the sum of two exponentials with time constants $\tau_{1}$ and $\tau_{2}$ and amplitudes $A_{1}$ and $A_{2}$ where

$$
U=A_{1} \times \exp \left(-p t / \tau_{1}\right)+A_{2} \times \exp \left(-p t / \tau_{2}\right) .
$$

Except for a time scaling factor, $p$, this function determines what fraction of the initial fluorescence remains at each time point, provided that fluorescence declines because of bleaching alone. To calculate the bleaching curve for a coverslip subjected to field stimulation, the fluorescence readings from the coverslip were averaged. Values during and after the stimulus were discarded, and remaining values were fitted to the product $C \times$ $U$, where $C$ is a constant. Aside from $C$, the only free parameter was $p$, the variable required to reconvert the unit of time in Equation 1 to seconds. An example is given in Figure 2D. Traces were first corrected for background and then for bleaching.

Analysis was done in Excel, and curve fitting was done in Origin (Microcal Software, Northampton, MA). Fluorescence values are given in analog-to-digital conversion units of our camera [arbitrary units (a.u.)] unless indicated otherwise.

Calibration. During most experimental sessions, fluorescent beads were imaged under the same conditions as neurons $[0.87 \mu \mathrm{m}$ diameter coated with $980 \pm 15$ fluorescein isothiocyanate (FITC) molecules; Bangs Laboratory, Fishers, IN] (Aravanis et al., 2003). Beads were located under DIC with "Find Spots" (1.27 $\mu \mathrm{m}$ circles). Circular regions surrounding single, solitary beads were selected and transferred into the epifluorescence image. Background was measured for each circle with a concentric annulus of $1.27 \mu \mathrm{m}$ inner and $2.86 \mu \mathrm{m}$ diameter

In one experiment, the experimental chamber was filled with FITC (0.1 $\mu \mathrm{M}$ in Tyrode's solution) or FM1-43 [0.1 $\mu \mathrm{M}$ in 1\% CHAPS (3-[(3cholamidopropyl)dimethylammonio]-1-propanesulfonate)] (Henkel et al., 1996). The microscope objective was focused first on the glass coverslip, and then the focus was moved into the solution by a distance of 40 $\mu \mathrm{m}$. Measurements with Tyrode's solution were subtracted as background.

\section{Results}

Synaptic vesicles in neurons may be stained selectively with FM1-43 (Betz and Bewick, 1992; Cochilla et al., 1999), an amphipathic molecule that fluoresces strongly when it intercalates between the lipid molecules of biological membranes. Synaptic boutons containing multiple stained vesicles fluoresce brightly, and the release of dye from them is readily recorded as synaptic vesicles undergo exocytosis. To study the behavior of single vesicles, we stained only one or a few vesicles per bouton (Ryan et al., 1997, Murthy and Stevens, 1998; Zenisek et al., 2000; Aravanis et al., 2003; Lemke and Klingauf, 2005) by taking advantage of the slow spontaneous recycling of synaptic vesicles during a 1 min exposure to FM1-43 (Ryan et al., 1997). Spontaneous and stimulated recycling both load the same pool of synaptic vesicles, because (1) stimulation causes synapses to destain in a $\mathrm{Ca}^{2+}$-dependent manner and with the same kinetics no matter how they were loaded, and (2) the rates of destaining vary coordinately among synapses in the two staining methods (Prange and Murphy, 1999; Groemer and Klingauf, 2007). Spontaneous recycling in addition loads a pool of vesicles that does not readily destain in response to electrical stimulation (Sara et al., 2005), but the short exposures used here are not expected to have labeled that pool.

Figure $1 A$ shows a typical image, showing one thick fascicle of axons and dendrites as well as thinner ones. The fascicles contain diffuse fluorescence with superimposed fluorescent dots presumably representing individual stained presynaptic boutons. During a period of field stimulation, all spots dimmed to some degree, and some disappeared completely (compare Figs. $1 B, C$ ). To detect the local release of dye, spots were located in images such as Figure $1 B$ by an automatic algorithm and then selected. Of the 524 spots originally found in the experiment of Figure 1, 407 were rejected as unsuitable for the study of single vesicles because they were oblong, had close neighbors (asterisks in Fig. $1 \mathrm{~B}$ ), or for other reasons (see Materials and Methods). We assumed all remaining 117 spots to represent presynaptic boutons. Some fluorescent boutons in our culture were mobile (Betz et al., 1992; Fischer et al., 1998; Krueger et al., 2003; Lemke and Klingauf, 2005; Darcy et al., 2006), and their sideways movement would lead to a local loss in fluorescence that could be mistaken for exocytosis. To recognize such movements, we added a constant to all pixels in images as in Figure $1 B$ and then subtracted an image taken at the end of our analysis period (Fig. 1C). The resulting difference image showed a gray background on which darkness marked a local gain and brightness a local loss in fluorescence. A gain can result only from movement, and spots with shadows (arrows in Fig. 1D) were not analyzed further.

\section{Quantal dye release}

We determined the time course of fluorescence while a $1 \mathrm{~s}$ train of electrical shocks was applied to the coverslip to generate action potentials. Shocks were given at high frequency $(100 \mathrm{~Hz})$ to increase the chance of observing exocytosis. Figure 2, $A$ and $B$, shows representative examples (boutons 1 and 2 in Fig. 1). The majority of spots dimmed continuously over the period analyzed (Fig. 2 A), presumably because of bleaching. A minority (32 of 117) also dimmed abruptly (Fig. $2 B$ ) and did so nearly always during the period of stimulation (26 of 32 in Fig. 2). In otherwise identical experiments without field stimulation, downward steps almost never occurred (3 of 420 traces as in Fig. 2 B, 6 coverslips).

For analysis, we first averaged the fluorescence in cell-free regions (Fig. 2C) as background. Then we determined the average time course of bleaching (Fig. $2 D$ ) in recordings without stimulation (see Materials and Methods). Figure 2, E and $F$, replots the traces in $A$ and $B$ after first subtracting the background and then dividing by the bleaching curve. The traces appeared nearly flat except where $F$ showed an abrupt fluorescence drop. Other boutons from this cell released dye incompletely $(G)$, in larger amounts $(H)$, or in multiple steps $(I)$. Trace $J$, finally, shows the average from $\sim 1000$ regions in this and other experiments and demonstrates that FM1-43 was released specifically while stimulation was in progress. Fluorescence failed to decline to background levels, because the stimulation stopped before most boutons could respond and because some boutons lost only a fraction of their fluorescence (Fig. 2G). The decline in Figure $2 J$ appeared gradual, as it did in earlier work exploring the release from hippocampal synapses containing tens of stained vesicles (Ryan et al., 1993; Klingauf et al., 1998).

Like others who have explored the quantal nature of synaptic vesicle turnover with FM1-43, we measured the loss of dye $(\Delta F)$ by comparing in each spot the fluorescence before and after stimulation (Fig. $2 K$ ). The resulting histogram is bimodal or trimodal and provides evidence of two or three populations. The first represents inactive boutons and includes results from traces as in Figure $2 E$ with no fluorescence loss other than what is expected from bleaching. The second and third peaks result from spots with an additional fluores- 
A

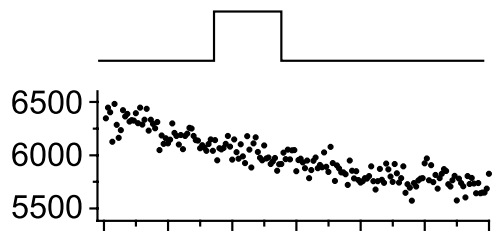

B

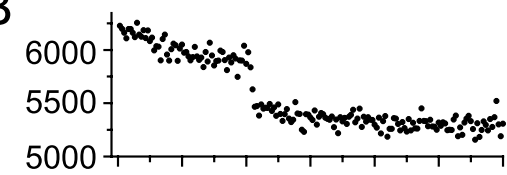

C

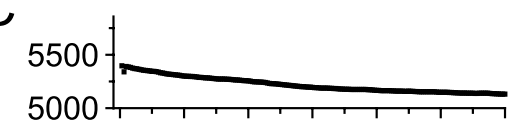

D

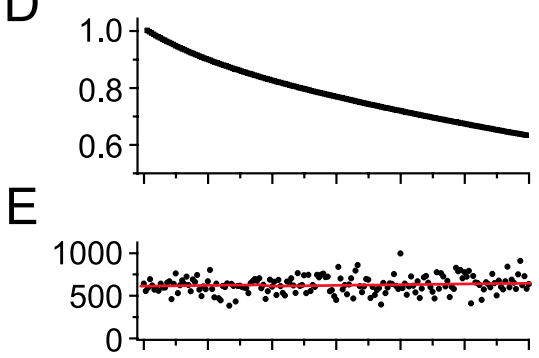

F

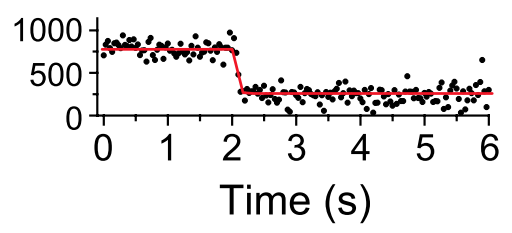

G

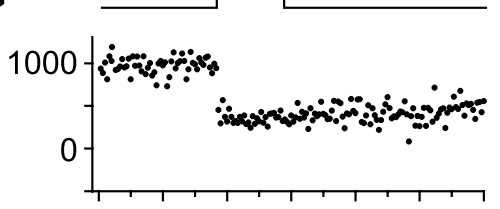

$\mathrm{H}$

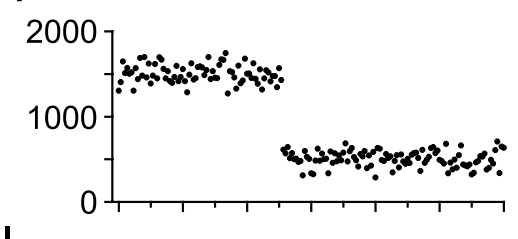

I
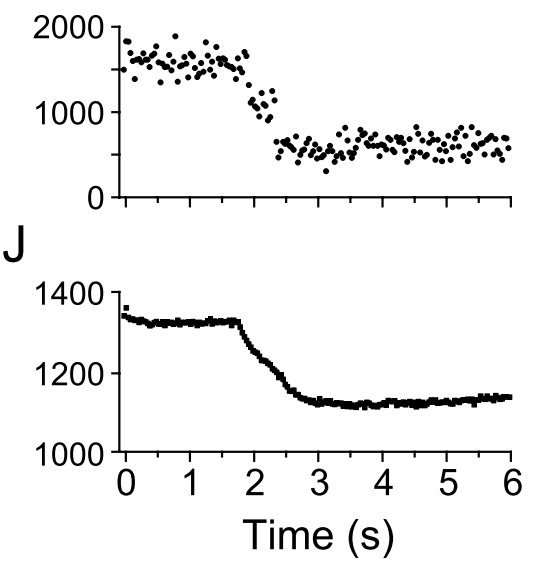

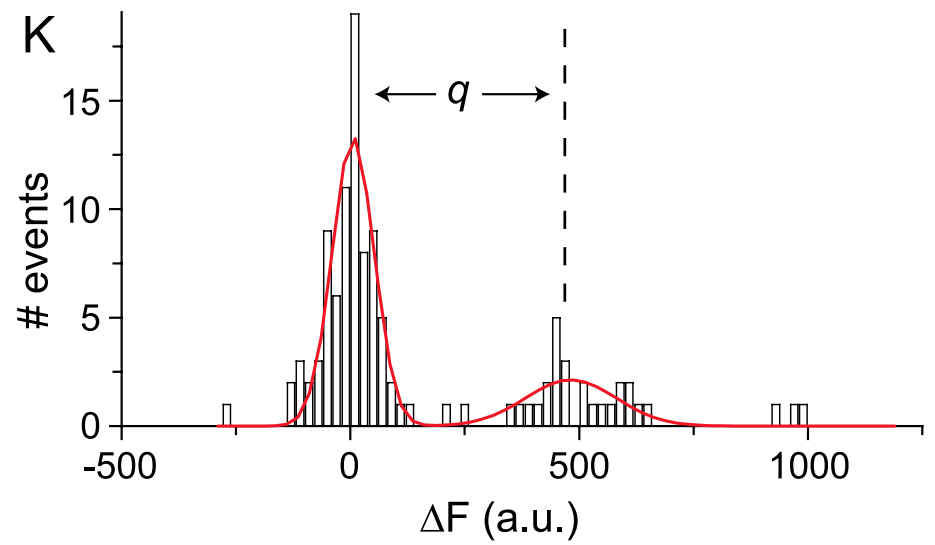

Figure 2. Stepwise fluorescence changes. The topmost line shows the timing of field stimulation (1 s at $100 \mathrm{~Hz}$ ). $\boldsymbol{A}, \boldsymbol{B}$, Fluorescence in the regions marked 1 and 2 in Figure $1 B$, measured in each frame of a $28 \mathrm{~Hz}$ movie and plotted as a function of time. $C$, Background fluorescence in cell-free regions ( $n=24$ in this experiment). It does not include the faint and diffuse glow of the fascicles over which most fluorescent spots are superimposed and underestimates the background. $\boldsymbol{D}$, Bleaching curve (see Materials and Methods). $\boldsymbol{E}$ and $\boldsymbol{F}$ are traces $\boldsymbol{A}$ and $\boldsymbol{B}$ after first subtracting curve $\boldsymbol{C}$ and then dividing the result by the curve in $\boldsymbol{D}$. The red lines in $\boldsymbol{E}, \boldsymbol{F}$, and $I$ are given by Equation 2 , where $b, m, t_{e,} S$, and $\tau$ were adjusted to obtain the bestfit. The constraints were that $0<\tau<0.1$ sand that $t_{e}$ falls between $t=1.95$ and $3.1 \mathrm{~s}$, a period including the frames during which stimulation occurred. The fit was sensitive to the initial choice for the parameters, especially for $t_{e}$. We set $b$ initially to the average of the first 10 data points, ran the fitting routine three times with initial values of $1.9,2.5$, and $3.1 \mathrm{~s}$ for $t_{e}$, and chose the result with the smallest $\chi^{2}$ value. In $\boldsymbol{E}$, the amplitude $S$ was so small (34 units, $t_{e}=2.5 \mathrm{~s}$ ) that no step is visible at this magnification. $\mathbf{G}-\boldsymbol{I}$, From other regions analyzed as in $\boldsymbol{E}$ and $\boldsymbol{F}$. In $\boldsymbol{I}$, the fit was poor, and, with steps separated by longer intervals, the fit picked out one of the two steps and ignored the other.J, Average from 1083 spots in this and 10 other coverslips, analyzed as in $\boldsymbol{E}$ and $\boldsymbol{F}$. $\boldsymbol{K}$, Release of dye during stimulation in all 117 boutons selected on this coverslip, plotted as a histogram. In each bouton, the fluorescence loss $(\Delta F)$ was determined as the difference between fluorescence averages of the last 30 frames before and of frames 5 to 35 after the last shock. The curve represents the sum of two Gaussian functions with peaks separated by a distance $q$, the quantal size in this coverslip ( 476 units in the fit). All ordinates except in $\boldsymbol{K}$ give fluorescence in arbitrary units. Data are from the same experiment as Figure 1.

cence loss. Multimodal histograms with evenly spaced peaks indicate that dye was lost in packets of uniform size and that dye uptake or release or both were quantal. Similar histograms were obtained previously when others gave more shocks to stimulate release during longer periods (Ryan et al., 1997; Murthy et al., 1998; Aravanis et al., 2003; Lemke and Klingauf, 2005).

The average size, $q$, of the quantum was determined for each coverslip by fitting the sum of two Gaussian functions and measuring the distance between their peaks (mean $q=461 \pm 30$ a.u., $n=11$ ). The region analyzed on each coverslip typically contained one to three cell bodies; therefore, the value of $q$ is derived from one to three cells. To place the value of $q$ on a molecular scale, we first measured the fluorescence of beads having the approximate size of a synaptic bouton and carrying an average 980 fluorescein (FITC) molecules on their surfaces (Aravanis et al., 2003). The mean was 3055 a.u., hence $q$ corresponds to $461 / 3055=0.155$ times the fluorescence of a bead, or 151 FITC molecules. There was variation (range of 105-226 FITC equivalents in this dataset) when coverslips were analyzed individually, and some of it may reflect genuine variation between cells or cultures in the amount of dye taken up by a vesicle.

When solutions of FITC $(0.1 \mu \mathrm{M}$ in Tyrode's solution) and FM1-43 (0.1 $\mu \mathrm{M}$ in $1 \%$ CHAPS) were examined under our microscope, FITC was 1.6 times brighter than FM143. If the dye is as bright in the membrane of synaptic vesicles as it is in CHAPS, then $q$ corresponds to the release of $151 \times 1.6=242$ molecules when boutons were stained with 10 $\mu \mathrm{M}$ FM1-43.

Experiments as in Figure 2 were also performed on cultures loaded with dye at other concentrations. The value of $q$ was $162 \pm 11$ FITC molecules at $5 \mu \mathrm{M}$ ( $n=5$ coverslips) and $192 \pm 19$ FITC molecules at $16 \mu \mathrm{M}(n=7$ coverslips). It varied less than proportionally with concentration, consistent with the in vitro result that concentrations in the range explored here nearly saturate the cytoplasmic leaflet of synaptic vesicle membrane (Richards et al., 2005).

Our minimally stained boutons rarely released more than two labeled vesicles. To verify that our cultures could be stained more vigorously, FM1-43 was applied while 50 shocks were given. Coverslips were much brighter than after minimal staining and lost $3954 \pm 305$ FITC units $(n=19$ coverslips with 70-250 boutons each) while they destained in response to 500 shocks $(10 \mathrm{~Hz})$. With $q=151$ FITC units, each bouton released $26 \pm 2$ quanta, a number comparable 
with a previous measurement on maximally loaded boutons (29 quanta) (Aravanis et al., 2003).

\section{Single fusion events}

Our results confirm that boutons release dye in packets of similar size. The downward steps also tell when each packet is released. To determine the amplitudes of steps, $S$, and the times of their occurrence, $t_{e}$, traces as in Figures $2 E-I$ were fitted with declining exponentials (red in Fig. $2 E, F$ ):

$$
\begin{array}{cc}
F=b+m t \quad & \text { for } t<t_{e} \\
F=b+m t-S \exp \left[-\left(t-t_{e}\right) / \tau\right] \\
\text { for } t>t_{e},
\end{array}
$$

where $F$ is the fluorescence, $t$ is time, and $b$, $m$, and $\tau$ are constants. Equation 2 applies if a fusion occurring at time $t_{e}$ releases $S$ fluorescence units with a time constant $\tau$; the sloping line $m t$ is included to allow for changes unrelated to fusion. We asked to what degree the fluorescence loss $(\Delta F$ in Fig. $2 K)$ is accounted for by downward steps when $S$ is based on fits with $\tau<0.1 \mathrm{~s}$ in Equation 2. The two measurements are highly correlated, and most fall on a line with a slope close to unity (Fig. 3A). Evidently, steps accounted entirely for the fluorescence loss in this cell. Moreover, the steps occurred specifically during the stimulation period, with the exact time of occurrence scattered throughout that period (Fig. 3B).

Figure $3 C$ plots data from this and similar experiments. With its two peaks, the histogram clearly distinguishes inactive from active boutons. Nonetheless, we wondered whether the second peak was artifactually broad because of day-to-day variations in our cultures or the intensity of illumination. To allow for such variations, step amplitudes were divided by the value of $q$ obtained for each coverslip as in Figure $2 \mathrm{~K}$. With all step amplitudes combined, the histogram is no less broad than in Figure $3 C$ and, in particular, still shows a "tail" comprising boutons releasing significantly more dye than others. The data are well described by a quantal model (Fig. 3D) wherein boutons released zero, one, or two stained vesicles. The second peak is at $S / q=$ 0.97 , supporting the idea that the fluorescence lost during stimulation is released in steps.

How variable is the amount of dye released in single fusion events? Because the first peak results from inactive boutons, its width estimates the error of the measurement. The second peak is broader than the first, and the additional variance represents the coefficient of variation for the amount of dye released during individual fusions. Because synaptic vesicles are expected to take $=0.20 \pm 0.02$
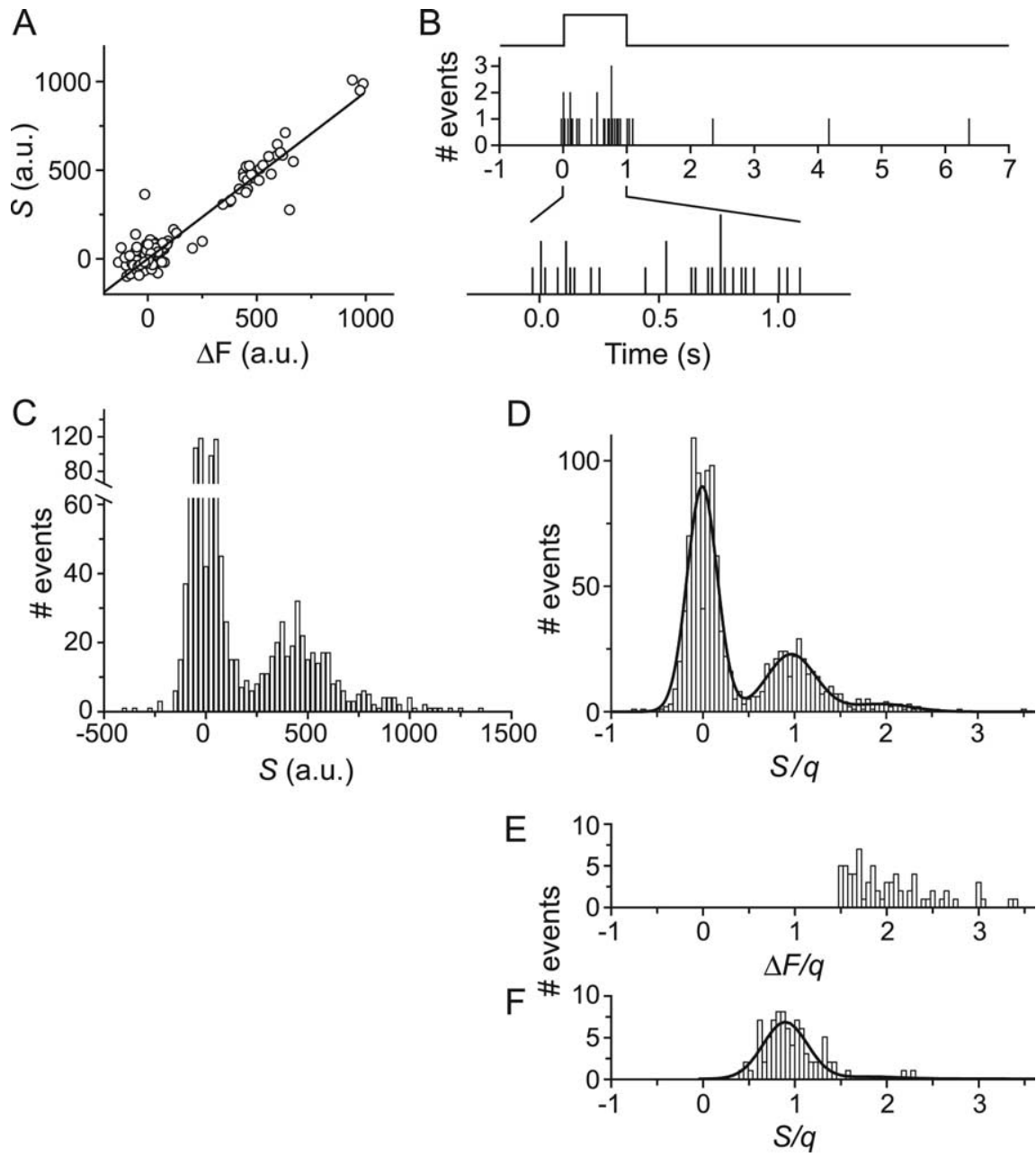

Figure 3. Single fusion events account for dye release. $A$, Comparing two methods for measuring dye release in the experiment of Figure 2. Abscissa, $\Delta F$; ordinate, amplitude $S$ of exponentials fitted to traces as in Figure 2, $E$ and $F$. The regression line through the origin has a slope of 0.94 . $B$, Histogram of latencies ( $t_{e}$ values) of all steps with amplitudes $>300$ fluorescence units in Figure 2; abscissa gives time after the onset of the stimulation period. Unlike in $\boldsymbol{A}$, no limits were placed on the value of $t_{e}$ in Equation 2. C, Step amplitude histogram from the experiment in Figures 1 and 2 as well as 10 other coverslips $(n=1083$ boutons). $\boldsymbol{D}$, Step amplitudes in $\boldsymbol{C}$ divided by the value of $q$ obtained as in Figure $2 \mathrm{~K}$ for each coverslip. The curve is a sum of Gaussians representing a quantal model adapted from Boyd and Martin (1956) and Murthy and Stevens (1998), wherein boutons release anywhere from zero to three aliquots or quanta of dye. The peak near the origin represents boutons failing to release dye, and the other, progressively smaller peaks represent boutons releasing one quantum or more:

$y(S)=A_{0} \exp \left[-0.5(S-c)^{2} / w_{m}{ }^{2}\right]+\sum A_{i} \exp \left[-0.5(S-c-i r)^{2} /\left(w_{m}{ }^{2}+i w_{r}{ }^{2}\right)\right]$.

The first peak has the amplitude $A_{0}$ and the position $c$. Its $S D$ is $w_{m}(0.093$ in the fit) and is taken to reflect measurement uncertainty. The remaining terms $(i=1-3)$ represent Gaussians of amplitudes $A_{i}$ spaced as integral multiples of the mean quantal size, $r$. Their variances are sums of $w_{m}$ with integral multiples of $w_{r}$, where $w_{r}$ is the coefficient of variation of the quantal size when $r=1$, as in this case. All variables are free parameters. The expected value of $c(-0.016$ in the fit) is 0 , and that of $r$ $(0.97 \pm 0.04$ in the fit) would be unity if the quantal sizes determined from step amplitudes equaled those determined from the fluorescence loss during the stimulation period. The value of $w_{r}=0.21 \pm 0.05 . E, \Delta F$ values as in Figure $2 K$ were divided by $q$, and values with $\Delta F / q>1.5$ plotted as a histogram. $\boldsymbol{F}$, Step amplitudes during multiple fusions. First, the traces on which $\boldsymbol{E}$ is based were fitted with Equation 2 to determine $\tau$. Next, traces with $\tau>0.2$ s were refitted with two (Eq. 4) or three exponentials occurring in succession; $\tau$ set to $50 \mathrm{~ms}$. When multiple exponentials fitted better than one, their amplitudes were included in the histogram. The curve represents Equation 3 with $A_{0}, A_{3}$ set to 0 and $w_{m}$ set to 0.093 . The fit resulted in $r=0.90 \pm 0.01$ and $w_{r}$

up dye in proportion to their surface area, the additional variance (0.23) translates into a coefficient of variation of 0.11 for the vesicle diameters on a given coverslip. A similar value of 0.1 was obtained by electron microscopy (Schikorski and Stevens, 1997). Hence, the release of dye during individual fusions is no more variable than the surface areas of synaptic vesicles.

Finally, we examined boutons losing $>1.5$ quanta of fluo- 
A

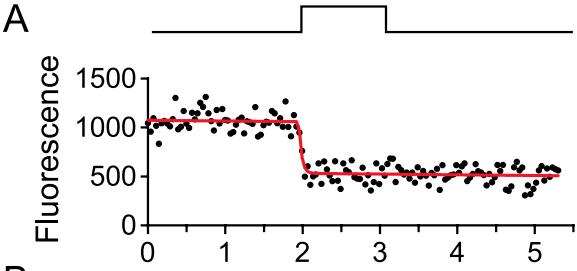

B

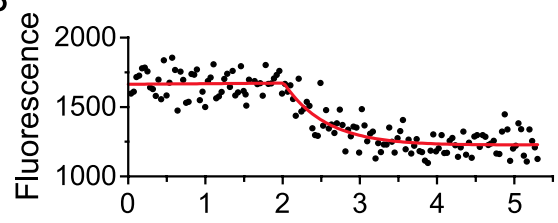

C

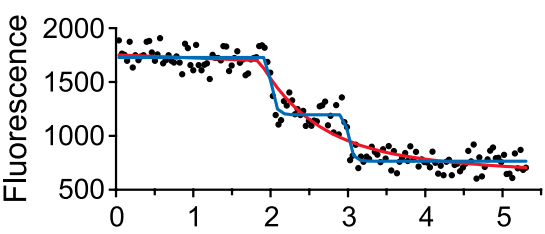

$\mathrm{D}$
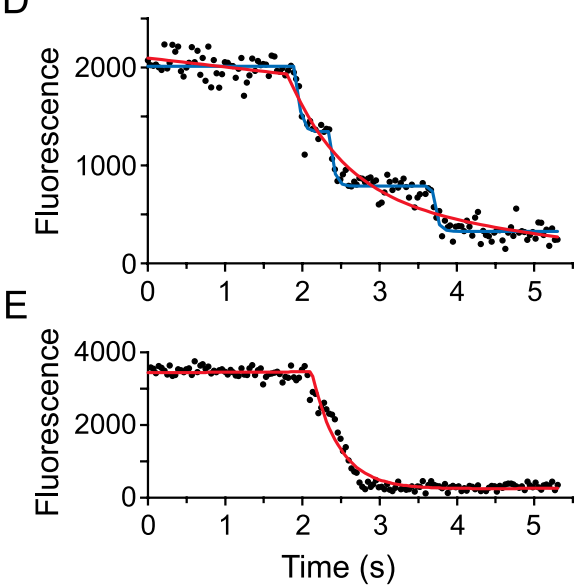
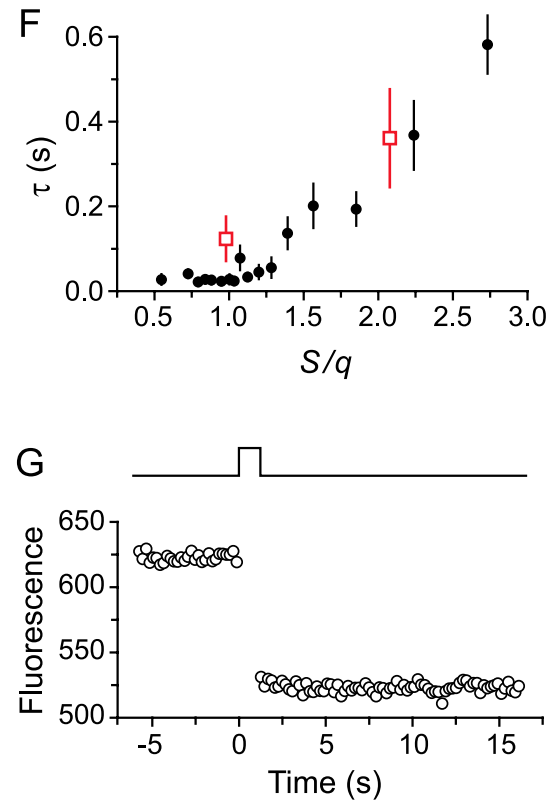

Figure 4. Time course of release. $A-D$, We selected all traces where $\Delta F>50$ FITC units, $\Delta F$ being the decline in fluorescence during the first $3 \mathrm{~s}$ after the beginning of the stimulus. Timing of the stimulus shown topmost. To each trace we fitted a straight line plus an exponential as in Figure $2, E$ and $F($ red), with $t, b, \tau, m$, and $S$ as free parameters. We set $b$ initially to the average of the first 10 data points, ran the fitting routine three times with initial values of $1.5,2.5$, and $4 \mathrm{~s}$ for $t_{e^{\prime}}$ and chose the result with the smallest $\chi^{2}$ value. The value of $t_{e}$ was constrained to $1.5<t_{e}<4.5 \mathrm{~s}$. When $S / q$ was between 0.75 and $1.25, A$ was the most frequent response and slower responses $(\boldsymbol{B})$ were rare. $\boldsymbol{C}$, Larger fluorescence changes usually were a sequence of downward steps. Red line, Single exponential with $\tau$ and $m$ in Equation 2 allowed to vary; the fit yielded $S / q=2, \chi^{2}=12,000$. Blue, Equation 4 with $\tau$ fixed at $0.05 s ; S_{1} / q=1.22$ and $S / q_{2}=0.96, \chi^{2}=8000$. D, Red as in $B$, but $S / q=2.53$ and $\chi^{2}=15864$. Blue as in Equation 4 with a third exponential term added, and $S_{1} / q=1.40, S_{2} / q=1.17, S_{3} / q=0.86$, and $\chi^{2}=9730 . E$, From an unusually bright bouton with an initial brightness of six times the quantal size. $F$, Time constant plotted against $S / q$. Black, From 346 traces as in $\boldsymbol{A}-\boldsymbol{D}$ from boutons loaded with $10 \mu \mathrm{m}$ FM1-43. Values were sorted by $\tau$ and then binned in packets of 20. Each point is the mean \pm SE of a bin. Red, Boutons loaded with $8 \mu \mathrm{M}$ FM1-84 ( $n=4$ coverslips). The two squares show means \pm SE for traces with $S / q$ between 0.75 and $1.25(n=33)$ and between 1.5 and $2.5(n=15)$. $G$, Absence of slow release of FM1-43 in $5 \mathrm{~Hz}$ movies (dataset in Fig. $5 E$ ) with the stimulus shown topmost. Average from 548 boutons in 10 cells. Thirty additional traces were not included because they showed downward steps beginning after the stimulus.

rescence (see histogram in Fig. 3E). Nearly half (32 of 79) released dye rapidly (Fig. $2 \mathrm{H}$ ), and recordings from them were well fitted by single-exponential functions with short time constants. The other half released dye more slowly and often in multiple steps. To identify slow boutons, recordings were fitted with a single exponential as in Equation 2 except that restrictions on the time constant $\tau$ were lifted. Of 79 recordings, 47 required $\tau>0.2 \mathrm{~s}$ and qualified as slow. They were next refitted with two (Eq. 4) or sometimes three exponentials starting in succession. To keep the number of free variables in the two fits comparable, $\tau$ was fixed at $0.05 \mathrm{~s}$ throughout. In 38 of the 47 boutons, two (Fig. $4 C$ ) or three successive exponential functions (Fig. $4 D$ ) fitted better than one, and amplitudes of the individual steps could be determined separately. When combined in a histogram (Fig. $3 F$ ), the amplitudes showed a distribution similar to the quantal peak in Figure 3D. Among the 79 boutons losing $>1.5$ quanta of dye, therefore, at least 38 did so in the form of additional quanta. In the remainder, most (32 of 41) released dye too fast to allow us to distinguish multiple steps if they existed, suggesting either that fusions followed each other too rapidly or that larger vesicles were released ( $\mathrm{He}$ et al., 2006). On the whole, our results show that most FM1-43 is released in packets of uniform size and that release occurs throughout stimulation in abrupt events. The simplest explanation is that the downward steps represent fusions of single synaptic vesicles.

\section{Time course of dye release from single and multiple vesicles}

Many downward steps went to completion within two successive frames, an interval indistinguishable from zero with a frame transfer camera. We wondered whether slower release occurred as well and examined all traces in the dataset of Figure 3C. Traces in which the fluorescence declined by $>50$ FITC equivalents were selected, regardless of whether or not the decline was stepwise or coincided with the time of stimulation. They were next fitted with a declining exponential function as in Figure 2, $E$ and $F$, except without constraints on $\tau$. Amplitude $S$ and time constant $\tau$ were determined. When $S / q$ was close to the mean quantal amplitude $(0.75<$ $S / q<1.25)$, the release was usually fast (Fig. 4A) with $\tau=34 \pm 4 \mathrm{~ms}(n=188)$. Slower release events were rare in that group ( $\tau>100 \mathrm{~ms}$ in 6\%); that in Figure $4 B$ was the slowest observed.

When $S / q$ was larger, the fit reported slower time constants (Fig. $4 F$ ). Among the 61 traces with $1.5<S / q<2.5,36$ required a time constant $>0.2 \mathrm{~s}$ but the fit was generally poor because the fluorescence declined in two steps (Fig. 4C). As an alternative, we fitted a modified Equation 2 with two exponential functions with amplitudes $S_{1}$ and $S_{2}$ and starting at $t_{e 1}$ and $t_{e 2}$ :

$$
\begin{array}{cc}
F=b & \text { for } t<t_{e 1} \\
F=b-S_{1} \exp \left[-\left(\mathrm{t}-\mathrm{t}_{\mathrm{e} 1}\right) / \tau\right] & \text { for } t_{e 1}<t<t_{e 2} \\
F=b-S_{1} \exp \left[-\left(t-t_{e 1}\right) / \tau\right]-S_{2} \exp \left[-\left(t-t_{e 2}\right) / \tau\right] \\
\text { for } t>t_{e 2} .
\end{array}
$$


With $\tau$ fixed at $0.05 \mathrm{~s}$, Equation 4 has the same number of free parameters as Equation 2, and the quality of fit by the two functions is readily compared. On the basis of $\chi^{2}$ values, the majority of traces (28 of 36) were better fitted with two exponentials (Eq. 4) than with one. With $S / q>2.5$, a third exponential term was sometimes required (Fig. 4D). We suggest that the fluorescence decline is generally stepwise under our conditions and looks graded (Fig. 4E) only when multiple, closely spaced steps blend into each other. Like Aravanis et al. (2003), we observed a tendency for second steps to be smaller than the first $\left(S_{2} / S_{1}=\right.$ $0.92 \pm 0.06, n=38, p<0.05$ in paired $t$ test), but the difference was small. Possibly, smaller synaptic vesicles fuse more reluctantly than larger ones.

The styryl dye FM1-84 [N-(3-triethylammoniumpropyl)-4-(4-dipentylamino)styryl) pyridinium dibromide] is identical to FM1-43 except for two additional $\mathrm{CH}_{2}$ groups, and its higher affinity for membranes may lead to a slower desorption into the aqueous phase. The two dyes have been compared in previous work. When they were applied to neurons and rapidly washed out, FM1-43 was lost from neuronal plasma membrane about twice slower than FM1-84 (Ryan et al., 1996; Klingauf et al., 1998), but when synaptic vesicles were stained and then destained by field stimulation, the difference between the two dyes was small (Klingauf et al., 1998) or absent (Ryan et al., 1996). When we investigated the destaining of single vesicles, results with FM1-84 were similar to those with FM1-43. As with FM1-43, a fit of Equation 2 reported longer time constants for the boutons releasing larger amounts of dye (red in Fig. $4 F$ ). As with FM1-43, such boutons generally released dye in multiple steps. When the loss of fluorescence was small $(0.75<S / q<1.25)$, the mean time constant was larger for FM1-84 $(\tau=123 \pm 52 \mathrm{~ms}, n=35$ boutons in 4 cells) than for FM1-43, but the difference did not reach statistical significance.

Finally, we asked whether there is a population of vesicles releasing dye with time constants of multiple seconds, as was observed previously when exocytosis was stimulated by elevated external $\left[\mathrm{K}^{+}\right]$(Richards et al., 2005). Boutons were imaged on a slower timescale, stimulated for $1 \mathrm{~s}$, and corrected for bleaching as before (see Materials and Methods). The average fluorescence shows little or no sign that vesicles fusing during the stimulus continued to release dye once the stimulus was over (Fig. 4G). Hence, the overwhelming majority of vesicles released dye rapidly under the conditions we used.

\section{Fluorescence of mobile synaptic vesicles}

Single exocytic events of the size observed here were previously attributed to the partial release of FM1-43 from vesicles undergoing kiss-and-run exocytosis (Aravanis et al., 2003). Although kiss-andrun is not favored at the high stimulation frequencies used here
(Harata et al., 2006), it seemed nonetheless possible that the events we observed reflected partial release. To estimate the dye content of a vesicle independently of exocytosis, we took advantage of the fact that, at any given time, a fraction of synaptic vesicles is in transit along axons. Clusters of vesicles detach from active zones, travel down the axon to join other active zones, or form new "orphan" release sites that, when stimulated, release FM1-43 with the same kinetics as synaptic boutons (Krueger et al., 2003; Darcy et al., 2006). The minimal staining protocol used here causes only one or few vesicles per bouton to be stained, and the same should be true for traveling clusters. Traveling structures were readily observed. The traveler in Figure 5A first moved downward, later reversed course, and finally came to rest within a circle (drawn in yellow). Movement made the structures easy to detect even when they were dim; that in Figure $5 B$ was the dimmest we observed. In Figure $5 C$, the fluorescence of travelers, $\Delta F$, was determined from the difference of values measured before and after the vesicle had arrived in the circle. The histogram plotting the $\Delta F$ values from Figure $5 C$ is well fitted by a quantal model, consistent with the idea that traveling clusters contained less than four stained vesicles, each containing 99 FITC equivalents of dye (Fig. 5D). 
A
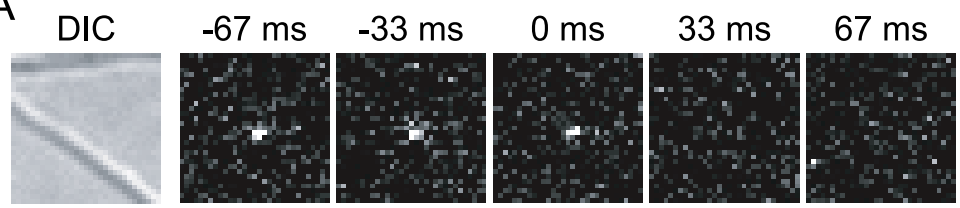

B
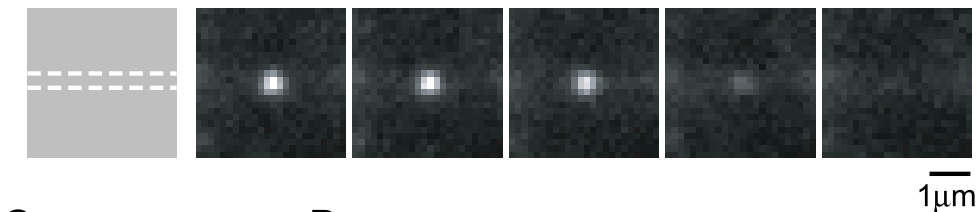

C

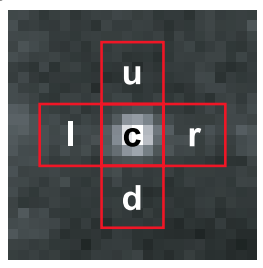

D

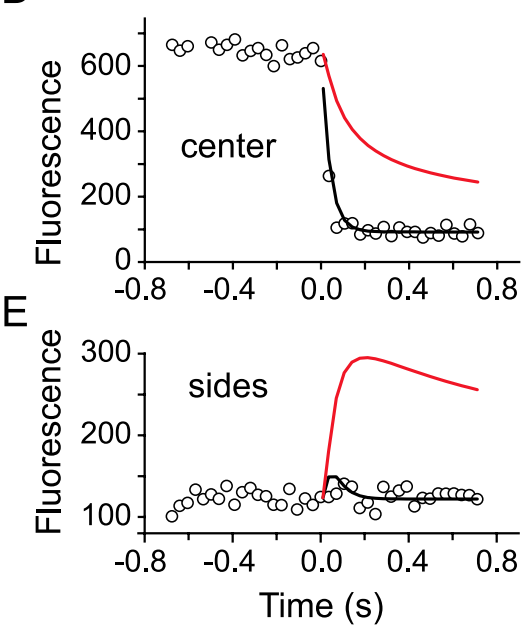

Figure 6. Exocytosis adds little or no FM1-43 to the axon. $\boldsymbol{A}$, Axon or small fascicle observed with DIC (leftmost) and with fluorescence. Middle, Section of a movie centered on a solitary stained bouton abruptly losing its dye. Times are relative to the last frame showing the bouton at full brightness and give the beginning of each exposure. Rightmost, Average of movie with the axon dimly visible. $\boldsymbol{B}$, We selected 55 boutons that destained completely and in a single step. Movies were temporally aligned such that they contained 20 frames immediately before and 20 frames after the bouton started to dim. The movies were then rotated such that the axon appeared horizontal, as judged under DIC (shown schematically on left), and finally averaged. A section of the average is shown, with times as in $\boldsymbol{A}$. C, Magnified from Figure $6 B$ with regions outlining the bouton ( $c$ ), portions of the adjoining axon $(I, r)$, and empty coverslip above and below $(u, d)$. The average of $u$ and $d$ was taken to represent the background close to the bouton. Region $\boldsymbol{C}(\boldsymbol{D})$ and average of regions / and $r(\boldsymbol{E})$ were both corrected for background but not bleaching. Curves in $\boldsymbol{D}$ show predictions for averages in the region c from diffusion in an infinitely long cylinder (Crank, 1980, their Eq. 2.6). Dye is assumed to be concentrated at a point within the cylinder at time 0 and then to diffuse away with a diffusion coefficient of $1.2 \mu \mathrm{m}^{2} / \mathrm{s}$, the value measured for FM1-43 in goldfish bipolar cells (Zenisek et al., 2002). Dye either remains in the plasma membrane (red) or departitions into the external space with a time constant of $46 \mathrm{~ms}$ (black). Curves in $\boldsymbol{E}$ as for $\boldsymbol{D}$ but for the average of regions $I, r$. The amplitudes of the calculated changes are overestimated if the diameter of the axon is less than that of the bouton.

During the movie, the cells on the coverslip were stimulated and the fluorescence of stationary boutons was plotted against time. As in Figure 2, boutons released dye in steps. Traces were fitted with a rapidly declining exponential whose amplitude was plotted as a separate histogram (Fig. 5E). The results are well fitted by a quantal model with a quantal size (95 FITC molecules in this dataset) that is indistinguishable from that in Figure 5D. A similar agreement was also observed in recordings at $28 \mathrm{~Hz}$ when travelers were analyzed as in Figure $5 A-D$ (data not shown). The quantal size was 138 FITC equivalents, similar to the step amplitudes obtained in that dataset (Figs. 2, 3). If vesicles released only a proportion of their dye during exocytosis, the quantal size for steps would have been smaller than for travelers. Evidently, vesicles empty during a fluorescence step.

\section{Little or no FM1-43 spreads into the axon}

When fish retinal bipolar neurons were observed with TIRF, FM1-43 was seen to spread into the surrounding plasma mem-

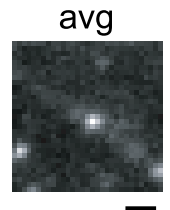

brane after exocytosis of synaptic vesicles (Zenisek et al., 2002). By analogy, one might expect FM1-43 to spread into the neighboring axon after exocytosis in hippocampal neurons. A test of this possibility is shown in Figure $6 \mathrm{~A}$ where the direction of the axon was determined under differential interference contrast. A fluorescent spot presumably representing a single stained vesicle darkened abruptly. To improve the signal-to-noise ratio by averaging, we selected movies showing boutons that had no close neighbors and lost essentially all their dye in a stepwise manner. Movies centered on the boutons were excised, temporally aligned, rotated to bring axons into a horizontal position, and then averaged (Fig. 6B). In the average movie, the fluorescence vanished abruptly as the vesicles underwent exocytosis (Fig. 6D) with no detectable spread into the axon to either side of the bouton (Fig. 6E). The results were compared with predictions for diffusion from a point source into an infinitely long cylinder, under two conditions. Under the first, the dye was assumed to desorb from membranes with a time constant of $46 \mathrm{~ms}$, similar to the average for boutons releasing a single quantum (black curves). Under the second, dye remained in the plasma membrane (red curves). The black curves are indistinguishable from the data, whereas the red curves do not fit. We conclude that most dye escapes from the bouton before it can diffuse into the axon.

The red curves would apply to TIRF recordings in which the cell surface tightly adheres to a glass and in which the external aqueous space is a thin layer sandwiched between the glass and the plasma membrane. A calculation may illustrate how effectively the glass prevents the dye from escaping from the membrane even if the off rate constant of the dye is infinite. Consider a spherical synaptic vesicle with internal diameter of $23 \mathrm{~nm}$ (Schikorski and Stevens, 1997) and a volume of $7 \times 10^{-6} \mu \mathrm{m}^{3}$. The aqueous interior of the vesicle will contain 0.02 dye molecules at $5 \mu \mathrm{M}$ [dye], and the membrane surrounding it will contain the rest. If each vesicle contains 250 molecules, $0.01 \%$ of the dye is dissolved in the interior of a vesicle. We assume that, during fusion, the vesicle flattens instantly into the plasma membrane, causing its internal surface of $0.0017 \mu \mathrm{m}^{2}$ to face the external aqueous space. If the thickness of that space is 0.05 $\mu \mathrm{m}$, dye must first fill an additional volume of $0.05 \times$ $0.0018=83 \times 10^{-6} \mu \mathrm{m}^{3}$. Although the increased aqueous space is now 14 times the volume of the synaptic vesicle, it will nonetheless contain only $0.1 \%$ of the dye of the vesicle. Dye also diffuses radially from the exocytic site, but no additional dye is lost into the space. Although diffusion dilutes dye, it does so by the same factor in both the aqueous space and the membrane such that neither will lose dye to the other. While diffusing in membrane closely apposed to glass, therefore, the 
vast majority of dye (99.9\% in this example) will remain in the membrane.

\section{Discussion}

Two steps in the synaptic vesicle cycle are intrinsically quantal, and styryl dyes can report them both. In the first, dye is sequestered when a vesicle buds from the plasma membrane, from larger membrane infoldings, or from stained endosomes (Miller and Heuser, 1984; Takei et al., 1996). In the second, dye is released from a synaptic vesicle during exocytosis. When hippocampal boutons were minimally stained with FM1-43 and then destained by exhaustive stimulation, the amount of dye released during destaining showed a quantal distribution (Ryan et al., 1997; Murthy and Stevens, 1998). To the extent that destaining was complete and individual fusion events were not observed, the quantum most likely reflected the sequestration or uptake of dye into the synaptic vesicle during the budding step (called uptake quantum hereafter). Uptake and release quantum should be equal in size, except in kiss-and-run exocytosis. Such equality was found by Murthy and Stevens (1998) but not confirmed when later work resolved single fusion events (Aravanis et al., 2003).

Here we imaged minimally stained boutons at high time resolution and under conditions of low background. Because vesicles have only a small chance of exocytosis during a single action potential, we stimulated with a brief ( $1 \mathrm{~s})$ train of shocks given at $100 \mathrm{~Hz}$. Although this frequency is higher than that of action potentials occurring physiologically, it facilitated detectable exocytosis in a narrow time window. In most stained boutons, fluorescence dimmed only because of photobleaching. However, those that released dye did so in discrete events marked by abrupt downward steps in fluorescence occurring randomly during the stimulation period. The steps fully accounted for the stimulusrelated loss of fluorescence, and their average size was equivalent to 145 fluorescein molecules. We do not know how strongly, relative to fluorescein, a single FM1-43 molecule fluoresces within the lipid bilayer of a synaptic vesicle. If both molecules fluoresce comparably, the number of molecules in a vesicle would be in the low hundreds. Given that frog motor neurons release 383 FM1-43 molecules per synaptic vesicle into the superfusion fluid (Henkel et al., 1996), the step size observed here is consistent with single synaptic vesicles.

Two findings support the idea that vesicles released dye in an all-or-nothing manner. First, the release was clearly quantal. Although the amount released varied somewhat from one fusion to another, it did so no more than the surface areas of synaptic vesicles observed by electron microscopy. For the release to be both quantal and incomplete, one would require that fusion pores have a uniform diameter and stay open for a fixed time, contrary to observations (Spruce et al., 1990; Curran et al., 1993; He et al., 2006). The second result is based on the fluorescence of minimally stained vesicle clusters traveling within axons. That fluorescence is also quantal and provides an estimate of the uptake quantum that does not depend on exocytosis. When measured in the same cells, uptake and release quanta were identical, contrary to expectation if vesicles released only part of their dye.

When care was taken to include only events resulting from the fusion of single vesicles, the time constant of release was $34 \mathrm{~ms}$. This is faster than washing the dye from the surface of neurons by superfusion (time constants 2-3 s) (Ryan et al., 1996; Klingauf et al., 1998) but slower than in stopped-flow experiments in which FM1-43 departitioned from the external (cytosolic) membrane leaflet of purified synaptic vesicles (time constant $7 \mathrm{~ms}$ ) (Richards et al., 2005). Our camera took $35 \mathrm{~ms}$ to acquire a single frame and cannot reliably measure a $7 \mathrm{~ms}$ time constant. Even with a faster camera, however, we would probably not see dye released as quickly as in a stopped-flow experiment. Synapses generally border on narrow spaces where FM1-43 may be caught in cycles of binding to, and unbinding from, the closely adjacent membranes of axons, dendrites, and glia. Perhaps the most striking example of unstirred spaces is the frog nerve terminal in which FM1-43 takes minutes to escape from cisternae formed after prolonged stimulation (Richards et al., 2000). Selecting thin axons and fascicles and imaging in the absence of glia will have lessened the problem of unstirred spaces but probably not eliminated it. In fact, more marked effects of unstirred spaces may explain the occasionally slower release of dye (Fig. $4 B$ ).

The following shows that release was too fast to be mediated by fusion pores of known properties. Recently, the fusion of single synaptic vesicles was investigated by capacitance measurements in the calyx of Held (He et al., 2006). Approximately $20 \%$ of vesicles underwent kiss-and-run and, for $270 \mathrm{~ms}$, maintained a fusion pore wide enough to allow glutamate to escape with a time constant of $0.5 \mathrm{~ms}$. If a vesicle contains $230 \mathrm{FM} 1-43$ molecules, then only 1 in $10^{4}$ molecules are dissolved in the vesicular space and available to escape through the fusion pore. Even if the dye diffused through the fusion pore as fast as glutamate and bound and unbound from the vesicle membrane fast enough to remain at equilibrium during the release process, then the time constant for release would be $10^{4}$ times slower than for glutamate, or $5 \mathrm{~s}$. The release observed here was much faster. Either fusion pores dilated in milliseconds or dye escaped from vesicles directly into the plasma membrane. Together, our results are consistent with exocytosis resulting in full fusion. They are also consistent with "cavicapture" in which the vesicle cavity is retrieved, but only after styryl dyes have escaped from it. This was observed for dense-core granules (Taraska and Almers, 2004) and may occur also when an artificial "dense core" such as a quantum dot (Zhang et al., 2007) prevents synaptic vesicles from flattening into the cell surface.

Two previous studies reported the release from single vesicles in hippocampal neurons, and both found kiss-and-run to be the dominant form of exocytosis. Aravanis et al. (2003) determined the uptake quantum by analysis of fluorescence changes, $\Delta F$, under exhaustive stimulation. Boutons stimulated at $10 \mathrm{~Hz}$ destained slowly with a time constant of $10 \mathrm{~s}$. Because the amount of dye lost equaled one uptake quantum, the gradual decline was attributed to the summation of repeated fusions by one vesicle undergoing kiss-and-run. At $0.1 \mathrm{~Hz}$ stimulation, Aravanis et al. recorded fluorescence steps in boutons in which the size of the uptake quantum was known as well. The steps were smaller than one uptake quantum, once again indicating kiss-and-run. Aside from the speed of release after single fusions, the largest difference in our work is that steps generally were of the same size as both, the value of $\Delta F$ during near-exhaustive stimulation, and the uptake quantum determined in traveling vesicles. This applied even when boutons destained in multiple steps (Fig. 4C,D). $\Delta F$ in our work (192 FITC equivalents with $16 \mu \mathrm{M}$ FM1-43) was no larger than in the study by Aravanis et al. (500 FITC equivalents).

In a second study, two populations of steps were observed while neurons released FM1-43 asynchronously at elevated external $\left[\mathrm{K}^{+}\right]$(Richards et al., 2005). Large steps of uniform size were attributed to full fusions and were so fast $(\tau=0.6 \mathrm{~s})$ that they may not have been temporally resolved at the recording frequency used $(2.5 \mathrm{~Hz})$. Smaller steps were of variable size and slow $(\tau=$ $3 \mathrm{~s}$ ). Their smaller size was attributed to release being incomplete and their slowness to release occurring through a narrow fusion 
pore. Whether they instead represent multiple fusions was not tested. We did not study asynchronous events because we could not test their relationship to stimulation nor distinguish them from noise. Had stimulation caused slow release, there should have been a slow component in Figure $4 G$, but such component was small or absent. On the whole, the two papers and our work show clear differences, but they could be reconciled if kiss-andrun required gentler stimulation than that used here.

A distinction has been made between large and small presynaptic terminals or boutons (Harata et al., 2006). Large terminals have many active zones and $10^{4}$ to $10^{6}$ synaptic vesicles, thus are thought not to require kiss-and-run. Accordingly, there is little evidence for this mechanism at the frog neuromuscular junction (Richards et al., 2000) or in fish retinal bipolar neurons (Zenisek et al., 2002; Llobet et al., 2003), and, at the calyx of Held, kissand-run is relatively rare (He et al., 2006). In the hippocampus and elsewhere in brain, however, small boutons and varicosities predominate. The relatively few vesicles per bouton may require extensive reuse (but see Fernandez-Alfonso and Ryan, 2004) and may require kiss-and-run as the dominant form of exocytosis (Harata et al., 2006) (but see Granseth et al., 2006). The present results resemble those in retinal bipolar neurons (Zenisek et al., 2002). Both types of neurons were studied in the absence of glia and under strong stimulation. In both, the release of FM1-43 from individual vesicles was primarily complete and too fast to be resolved by video frequency imaging. We suggest that full fusion can be the dominant mechanism in both large and in small terminals.

\section{References}

Aravanis AM, Pyle JL, Tsien RW (2003) Single synaptic vesicles fusing transiently and successively without loss of identity. Nature 423:643-647.

Banker G, Goslin K (1998) Culturing nerve cells, Ed 2. Cambridge, MA: MIT.

Betz WJ, Bewick GS (1992) Optical analysis of synaptic vesicle recycling at the frog neuromuscular junction. Science 255:200-203.

Betz WJ, Bewick GS (1993) Optical monitoring of transmitter release and synaptic vesicle recycling at the frog neuromuscular junction. J Physiol (Lond) 460:287-309.

Betz WJ, Bewick GS, Ridge RM (1992) Intracellular movements of fluorescently labeled synaptic vesicles in frog motor nerve terminals during nerve stimulation. Neuron 9:805-813.

Boyd IA, Martin AR (1956) The end-plate potential in mammalian muscle. J Physiol (Lond) 132:74-91.

Cochilla AJ, Angleson JK, Betz WJ (1999) Monitoring secretory membrane with FM1-43 fluorescence. Annu Rev Neurosci 22:1-10.

Crank J (1980) The mathematics of diffusion. Oxford, UK: Oxford UP.

Curran MJ, Cohen FS, Chandler DE, Munson PJ, Zimmerberg J (1993) Exocytotic fusion pores exhibit semi-stable states. J Membr Biol 133:61-75.

Darcy KJ, Staras K, Collinson LM, Goda Y (2006) Constitutive sharing of recycling synaptic vesicles between presynaptic boutons. Nat Neurosci 9:315-321.

Fernandez-Alfonso T, Ryan TA (2004) The kinetics of synaptic vesicle pool depletion at CNS synaptic terminals. Neuron 41:943-953.

Fischer M, Kaech S, Knutti D, Matus A (1998) Rapid actin-based plasticity in dendritic spines. Neuron 20:847-854.

Granseth B, Odermatt B, Royle SJ, Lagnado L (2006) Clathrin-mediated endocytosis is the dominant mechanism of vesicle retrieval at hippocampal synapses. Neuron 51:773-786.

Groemer TW, Klingauf J (2007) Synaptic vesicles recycling spontaneously and during activity belong to the same vesicle pool. Nat Neurosci 10:145-147.

Harata NC, Choi S, Pyle JL, Aravanis AM, Tsien RW (2006) Frequency- dependent kinetics and prevalence of kiss-and-run and reuse at hippocampal synapses studied with novel quenching methods. Neuron 49:243-256.

He L, Wu XS, Mohan R, Wu LG (2006) Two modes of fusion pore opening revealed by cell-attached recordings at a synapse. Nature 444:102-105.

Henkel AW, Lubke J, Betz WJ (1996) FM1-43 dye ultrastructural localization in and release from frog motor nerve terminals. Proc Natl Acad Sci USA 93:1918-1923.

Heuser JE, Reese TS (1973) Evidence for recycling of synaptic vesicle membrane during transmitter release at the frog neuromuscular junction. J Cell Biol 57:315-344.

Klingauf J, Kavalali ET, Tsien RW (1998) Kinetics and regulation of fast endocytosis at hippocampal synapses. Nature 394:581-585.

Krueger SR, Kolar A, Fitzsimonds RM (2003) The presynaptic release apparatus is functional in the absence of dendritic contact and highly mobile within isolated axons. Neuron 40:945-957.

Lemke EA, Klingauf J (2005) Single synaptic vesicle tracking in individual hippocampal boutons at rest and during synaptic activity. J Neurosci 25:11034-11044.

Llobet A, Beaumont V, Lagnado L (2003) Real-time measurement of exocytosis and endocytosis using interference of light. Neuron 40:1075-1086.

Midorikawa M, Tsukamoto Y, Berglund K (2007) Different roles of ribbonassociated and ribbon-free active zones in retinal bipolar cells. Nat Neurosci 10:1268-1276.

Miller TM, Heuser JE (1984) Endocytosis of synaptic vesicle membrane at the frog neuromuscular junction. J Cell Biol 98:685-698.

Murthy VN, Stevens CF (1998) Synaptic vesicles retain their identity through the endocytic cycle. Nature 392:497-501.

Prange O, Murphy TH (1999) Correlation of miniature synaptic activity and evoked release probability in cultures of cortical neurons. J Neurosci 19:6427-6438.

Richards DA, Guatimosim C, Betz WJ (2000) Two endocytic recycling routes selectively fill two vesicle pools in frog motor nerve terminals. Neuron 27:551-559.

Richards DA, Bai J, Chapman ER (2005) Two modes of exocytosis at hippocampal synapses revealed by rate of FM1-43 efflux from individual vesicles. J Cell Biol 168:929-939.

Ryan TA, Reuter H, Wendland B, Schweizer FE, Tsien RW, Smith SJ (1993) The kinetics of synaptic vesicle recycling measured at single presynaptic boutons. Neuron 11:713-724.

Ryan TA, Smith SJ, Reuter H (1996) The timing of synaptic vesicle endocytosis. Proc Natl Acad Sci USA 93:5567-5571.

Ryan TA, Reuter H, Smith SJ (1997) Optical detection of a quantal presynaptic membrane turnover. Nature 388:478-482.

Sankaranarayanan S, Ryan TA (2000) Real-time measurements of vesicleSNARE recycling in synapses of the central nervous system. Nat Cell Biol 2:197-204.

Sara YT, Virmani T, Deak F, Lui XR, Kavalali ET (2005) An isolated pool of vesicles recycles at rest and drives spontaneous neurotransmission. Neuron 45:563-573.

Schikorski T, Stevens CF (1997) Quantitative ultrastructural analysis of hippocampal excitatory synapses. J Neurosci 17:5858-5867.

Spruce AE, Breckenridge LJ, Lee AK, Almers W (1990) Properties of the fusion pore that forms during exocytosis of a mast cell secretory vesicle. Neuron 4:643-654.

Takei K, Mundigl O, Daniell L, De Camilli P (1996) The synaptic vesicle cycle: a single vesicle budding step involving clathrin and dynamin. J Cell Biol 133:1237-1250.

Taraska JW, Almers W (2004) Bilayers merge even when exocytosis is transient. Proc Natl Acad Sci USA 101:8780-8785.

Zenisek D, Steyer JA, Almers W (2000) Transport, capture and exocytosis of single synaptic vesicles at active zones. Nature 406:849-854.

Zenisek D, Steyer JA, Feldman ME, Almers W (2002) A membrane marker leaves synaptic vesicles in milliseconds after exocytosis in retinal bipolar cells. Neuron 35:1085-1097.

Zhang Q, Cao YQ, Tsien R (2007) Quantum dots provide an optical signal specific to full collapse fusion of synaptic vesicles. Proc Natl Acad Sci USA 104:17843-17848. 